\title{
On Parameterized Stabilization of Networked Dynamical Systems
}

\author{
R. M. Hermans* M. Lazar* A. Jokic ${ }^{*}$ R. H. Gielen* \\ * Department of Electrical Engineering, Eindhoven University of \\ Technology, P.O. Box 513, 5600 MB Eindhoven, The Netherlands \\ E-mails: $\{$ r.m.hermans, m.lazar, a.jokic, r.h.gielen\}@tue.nl
}

\begin{abstract}
The problem of stabilizing networked dynamical systems (NDS) in a scalable fashion is addressed. As a first contribution, an example is provided to demonstrate that the standard NDS stabilization methods can fail even for simple linear time-invariant systems. Then, a solution to this issue is proposed, in which the controller synthesis is decentralized via a set of parameterized local functions. The corresponding stability conditions allow for max-type construction of a Lyapunov function (LF) for the full closed-loop system, while neither of the local functions is required to be a local LF. It is shown that the provided approach is nonconservative in the sense that it is able to find a stabilizing control law for the motivating example network, whereas state-of-the-art non-centralized Lyapunov techniques fail. For inputaffine NDS and quadratic parameterized local functions, the combined LF synthesis and control scheme can be formulated as a set of low-complexity semi-definite programs that are solved on-line, in a receding horizon manner.
\end{abstract}

Keywords: Lyapunov methods, Distributed control, Networked control systems, Large-scale systems

\section{INTRODUCTION}

Electrical power networks, urban water supply infrastructures, automated highways, coupled chemical processes and the Internet are but a few examples of systems that consist of a large number of interacting dynamical subsystems, see, e.g., (Strogatz, 2001; Camponogara et al., 2002; Dunbar, 2007; Venkat et al., 2008; Negenborn et al., 2008) and the references therein. Many of such networked dynamical systems (NDS) are characterized by significant nonlinearities, strict input and state constraints and, most importantly, coupling dynamics.

One of the challenges in NDS research is to establish asymptotic stability in a scalable way, which is crucial in the design of globally stabilizing control laws. Conventionally, stability is determined by verifying the existence of a quadratic Lyapunov function (LF), given a linearized description of the system dynamics. However, the large size of NDS (power systems, for instance, can have millions of states) renders this method infeasible, as the required full system model is usually difficult to obtain and the corresponding LF synthesis problem, formulated as a semidefinite program (SDP), comes with a computational burden that can be arbitrarily large.

The issues associated with the application of classical Lyapunov methods to large-scale NDS motivate the need for decentralizing the standard asymptotic stability conditions. Non-centralized stability analysis typically relies on dissipativity theory to generate a LF from a set of local storage and supply functions, see (Willems, 1972). Perhaps the simplest dissipativity-based stabilization methods re- quire linear system dynamics and employ fixed, quadratic storage functions and constant supply rates to obtain a separable set of SDP problems that are solved off-line, in a decentralized fashion. This includes approaches that require the supply functions to be zero for all the systems in the network, and consider storage functions that are local Lyapunov functions, see, e.g., (Sandell et al., 1978; Šiljak, 1991). Although decentralized methods are attractive because of their low complexity, they are often conservative, which significantly limits their applicability.

More advanced scalable Lyapunov methods aim to increase flexibility with respect to decentralized synthesis by employing state-parameterized supply functions that can be generated in a distributed fashion. As an example, we mention the scheme that was recently proposed in (Jokić and Lazar, 2009). This technique employs a set of parameterized supply rates and fixed, positive-definite storage functions, i.e., structured Lyapunov functions, the sum of which yields a Lyapunov candidate for the full network. However, the existing tractable, distributed methods for a-priori generating a set of quadratic structured LFs and supply rates for linear NDS, see, e.g., (Langbort et al., 2004), come with communication requirements that are incompatible with many large-scale applications. In (Hermans et al., 2010), a partial solution to this issue was provided that is based on "max-type dissipativity conditions". Such conditions do not require distributed optimization and allow for max-type construction of a LF, which provides more flexibility than the sum-type construction employed by the structured LF scheme, see also (Dashkovskiy et al., 2010). Even so, both the decentralized and distributed 
schemes described above suffer from the impediment that is characteristic to a-priori fixed storage function synthesis.

The contribution of this paper is twofold. First, we illustrate the conservatism associated with off-line LF synthesis by providing a motivating example of a controlled NDS that does not admit construction of a Lyapunov function from a set of fixed quadratic storage functions, but that is stable nonetheless. Then, the objective is to find a solution to this issue, by endowing the storage functions with a finite set of state-dependent parameters. Stability conditions similar to the ones provided in (Hermans et al., 2010) are employed to allow for max-type construction of a Lyapunov function for the full network, while neither of the parameterized storage functions is required to be a local LF. The merit of the proposed approach is that the parameterized local storage functions can be constructed during operation, i.e., along a trajectory, via a collection of coupled convergence conditions. Thus, the impediment of off-line synthesizing structured LFs via fixed storage functions is removed. The provided stability conditions are efficiently exploited in devising a non-centralized control scheme that relies on non-iterative communication among directly coupled systems only. For input-affine NDS and quadratic parameterized local functions, the combined synthesis and stabilization scheme can be implemented by solving a single low-complexity SDP problem at each node, in a receding horizon fashion. The motivating example is used to demonstrate that the concepts proposed in this paper may allow for stabilization in cases where conventional non-centralized methods based on off-line generated fixed quadratic storage functions and static state feedback fail.

\section{PRELIMINARIES}

Let $\mathbb{C}, \mathbb{R}, \mathbb{R}_{+}, \mathbb{Z}$ and $\mathbb{Z}_{+}$denote the sets of complex numbers, reals, non-negative reals, integers and non-negative integers, respectively. For each $c \in \mathbb{R}$ and $\Pi \subseteq \mathbb{R}$ we define $\Pi_{\geq c}:=\{k \in \Pi \mid k \geq c\}$ and similarly, $\Pi_{\leq c}$. Let $\mathbb{Z}_{\Pi}:=\mathbb{Z} \cap \Pi$. For a finite set $\left\{x_{i}\right\}_{i \in \mathbb{Z}_{[1, N]}}, x_{i} \in$ $\mathbb{R}^{n_{i}}, N \in \mathbb{Z}_{\geq 1}$, let $\operatorname{col}\left(\left\{x_{i}\right\}_{i \in \mathbb{Z}_{[1, N]}}\right)$ and $\operatorname{col}\left(x_{1}, \ldots, x_{N}\right)$ denote the vector $\left(x_{1}^{\top}, \ldots, x_{N}^{\top}\right)^{\top}$. Let $0_{n}$ be the zero vector in $\mathbb{R}^{n}$. For $x \in \mathbb{R}^{n}$, let $\|x\|$ be an arbitrary $p$ norm, i.e., $\|x\|_{p}:=\left(\sum_{i=1}^{n}\left|[x]_{i}\right|^{p}\right)^{\frac{1}{p}}$ for $p \in \mathbb{Z}_{[1, \infty)}$ and $\|x\|_{\infty}:=\max _{i=1, \ldots, n}\left|[x]_{i}\right|$, where $[x]_{i}, i \in \mathbb{Z}_{[1, n]}$, is the $i$-th component of $x$ and $|\cdot|$ is the absolute value operator. For a finite set $\left\{M_{i}\right\}_{i \in \mathbb{Z}_{[1, N]}}, M_{i} \in \mathbb{R}^{n_{i} \times m_{i}}, N \in \mathbb{Z}_{\geq 1}$, let $\operatorname{diag}\left(\left\{M_{i}\right\}_{i \in \mathbb{Z}_{[1, N]}}\right)$ be a $\sum_{i=1}^{N} n_{i}$ by $\sum_{i=1}^{N} m_{i}$ matrix with diagonal blocks $M_{i}$ and all-zero off-diagonal blocks. Let $\mathbf{z}:=\{z(l)\}_{l \in \mathbb{Z}_{+}}$with $z(l) \in \mathbb{R}^{n}, l \in \mathbb{Z}_{+}$, denote an arbitrary sequence. For a set $\mathcal{S} \subset \mathbb{R}^{n}$, let $\operatorname{int}(\mathcal{S})$ be the interior of $\mathcal{S}$. A function $\varphi: \mathbb{R}_{+} \rightarrow \mathbb{R}_{+}$is in class $\mathcal{K}$ if it is continuous, strictly increasing and $\varphi(0)=0 ; \varphi$ is in class $\mathcal{K}_{\infty}$ if $\varphi \in \mathcal{K}$ and $\lim _{s \rightarrow \infty} \varphi(s)=\infty$. A function $\beta: \mathbb{R}_{+} \times \mathbb{R}_{+} \rightarrow \mathbb{R}_{+}$is in class $\mathcal{K} \mathcal{L}$ if for all $k, s \in \mathbb{R}_{+}$, $\beta(\cdot, k) \in \mathcal{K}, \beta(s, \cdot)$ is decreasing and $\lim _{k \rightarrow \infty} \beta(s, k)=0$.

Consider the discrete-time autonomous nonlinear system

$$
x(k+1)=\Phi(x(k)), \quad k \in \mathbb{Z}_{+},
$$

where $x(k) \in \mathbb{R}^{n}$ is the state at discrete-time instant $k$ and $\Phi: \mathbb{R}^{n} \rightarrow \mathbb{R}^{n}$ is a nonlinear function. For simplicity, let the origin be an equilibrium of (1), i.e., $\Phi\left(0_{n}\right)=0_{n}$.
Next, suppose that $\mathbb{X} \subset \mathbb{R}^{n}$ contains an open neighborhood of $0_{n}$, and consider the following definition.

Definition 2.1. (i) System (1) is globally asymptotically stable $(G A S)$ if there is a $\mathcal{K} \mathcal{L}$-function $\beta(\cdot, \cdot)$ such that the trajectories of (1) satisfy $\|x(k)\| \leq \beta(\|x(0)\|, k)$ for all $k \in$ $\mathbb{Z}_{+}$and $x(0) \in \mathbb{R}^{n}$. (ii) System (1) is globally exponentially stable $(G E S)$ if it is GAS with $\beta(s, k):=c s \mu^{k}$, for some $c \in \mathbb{R}_{\geq 1}$ and $\mu \in \mathbb{R}_{[0,1)}$. System (1) is exponentially stable in $\mathbb{X}$, denoted by $\operatorname{ES}(\mathbb{X})$, if this property holds only for $x(0) \in \mathbb{X}$.

Theorem 2.2. (Jiang and Wang, 2001; Lazar, 2006). Let $\alpha_{1}, \alpha_{2} \in \mathcal{K}_{\infty}, \rho \in \mathbb{R}_{[0,1)}$ and $V: \mathbb{R}^{n} \rightarrow \mathbb{R}_{+}$satisfy

$$
\begin{aligned}
\alpha_{1}(\|x\|) \leq V(x) & \leq \alpha_{2}(\|x\|) \\
V(\Phi(x)) & \leq \rho V(x)
\end{aligned}
$$

for all $x \in \mathbb{R}^{n}$. Then (1) is GAS.

A function $V(\cdot)$ that satisfies the conditions of Thm. 2.2 is a Lyapunov function (LF) for system (1).

Consider a directed connected graph $\mathcal{G}=(\mathcal{S}, \mathcal{E})$ with a finite number of vertices $\mathcal{S}=\left\{\varsigma_{1}, \ldots, \varsigma_{N}\right\}$ and a set of directed edges $\mathcal{E} \subseteq\left\{\left(\varsigma_{i}, \varsigma_{j}\right) \in \mathcal{S} \times \mathcal{S} \mid i \neq j\right\}$. In a NDS, a dynamical system described by the difference equation

$$
x_{i}(k+1)=\phi_{i}\left(x_{i}(k), v_{i}\left(x_{\mathcal{N}_{i}}(k)\right)\right), \quad k \in \mathbb{Z}_{+},
$$

is assigned to each vertex $\varsigma_{i} \in \mathcal{S}$. Thus, $x_{i} \in \mathbb{R}^{n_{i}}$ is the state of system $i \in \mathcal{I}:=\mathbb{Z}_{[1, N]}$, i.e., the system assigned to vertex $\varsigma_{i}$. With each edge $\left(\varsigma_{j}, \varsigma_{i}\right) \in \mathcal{E}$ we associate a function $v_{i j}: \mathbb{R}^{n_{j}} \rightarrow \mathbb{R}^{n_{v_{i j}}}$ that defines the interconnection between systems $j$ and $i$, i.e., $v_{i j}\left(x_{j}(k)\right)$ characterizes how $x_{j}(k)$ influences the dynamics of system $i$. Let $\mathcal{N}_{i}:=\{j \mid$ $\left.\left(\varsigma_{j}, \varsigma_{i}\right) \in \mathcal{E}\right\}$ be the set of indices corresponding to the direct neighbors of system $i$. A direct neighbor of system $i$ is any system whose states/outputs appear explicitly (via $v_{i j}(\cdot)$ ) in the equations that govern the dynamics of system $i$. Clearly, if $j \in \mathcal{N}_{i}$, this does not necessarily imply that $i \in \mathcal{N}_{j}$. Let $\overline{\mathcal{N}}_{i}:=\mathcal{N}_{i} \cup\{i\}$. We define $x_{\mathcal{N}_{i}}(k):=\operatorname{col}\left(\left\{x_{j}(k)\right\}_{j \in \mathcal{N}_{i}}\right)$ as the vector that collects all the state vectors of the direct neighbors of system $i$ and $v_{i}\left(x_{\mathcal{N}_{i}}(k)\right):=\operatorname{col}\left(\left\{v_{i j}\left(x_{j}(k)\right)\right\}_{j \in \mathcal{N}_{i}}\right) \in \mathbb{R}^{n_{v_{i}}}$ as the vector that collects all the vector-valued interconnection signals that enter system $i$. The functions $\phi_{i}: \mathbb{R}^{n_{i}} \times \mathbb{R}^{n_{v_{i}}} \rightarrow \mathbb{R}^{n_{i}}$ and $v_{i j}(\cdot)$ satisfy $\phi_{i}\left(0_{n_{i}}, 0_{n_{v_{i}}}\right)=0_{n_{i}}$ for all $i \in \mathcal{I}$ and $v_{i j}\left(0_{n_{j}}\right)=0_{n_{v_{i j}}}$ for all $(i, j) \in \mathcal{I} \times \mathcal{N}_{i}$.

Finally, let the overall NDS dynamics be written in compact form as

$$
x(k+1)=\Phi(x(k)), \quad k \in \mathbb{Z}_{+},
$$

where $x=\operatorname{col}\left(\left\{x_{i}\right\}_{i \in \mathcal{I}}\right) \in \mathbb{R}^{n}, n=\sum_{i \in \mathcal{I}} n_{i}$, and where $\Phi: \mathbb{R}^{n} \rightarrow \mathbb{R}^{n}$ is defined as $\Phi(x):=\operatorname{col}\left(\left\{\phi_{i}\left(x_{i}\right)\right\}_{i \in \mathcal{I}}\right)$. From the assumptions on $\phi_{i}(\cdot)$ it readily follows that the origin is an equilibrium of (4), i.e., $\Phi\left(0_{n}\right)=0_{n}$.

\section{A MOTIVATING EXAMPLE}

Scalable Lyapunov methods play a key role in the design of non-centralized control laws for NDS. The existing techniques for establishing stability in a non-centralized way generally construct a LF from a set of fixed, quadratic storage functions that act on local state vectors only. The first contribution of this paper is to provide an example network to illustrate that these methods may fail, even for simple linear and time-invariant networks. 
Consider the following set of systems, interconnected as shown in Fig. 1 and having states and inputs $x_{i} \in \mathbb{R}^{2}$ and $u_{i} \in \mathbb{R}$, respectively, for $i \in \mathbb{Z}_{[1,4]}$ :

$$
x_{i}(k+1)=A_{i i} x_{i}(k)+\sum_{j \in \mathcal{N}_{i}} A_{i j} x_{j}(k)+B_{i} u_{i}(k),
$$

where $\mathcal{N}_{1}=\{2,3\}, \mathcal{N}_{2}=\{1,4\}, \mathcal{N}_{3}=\{2\}, \mathcal{N}_{4}=\{2\}$,

$$
A=\left[\begin{array}{llll}
A_{11} & A_{12} & A_{13} & A_{14} \\
A_{21} & A_{22} & A_{23} & A_{24} \\
A_{31} & A_{32} & A_{33} & A_{34} \\
A_{41} & A_{42} & A_{43} & A_{44}
\end{array}\right]=\left[\begin{array}{rr|rr|rr|rr}
-\frac{3}{4} & -1 & \frac{1}{2} & \frac{1}{4} & -\frac{1}{2} & \frac{1}{2} & 0 & 0 \\
\frac{1}{4} & 0 & \frac{1}{4} & 0 & 1 & \frac{1}{2} & 0 & 0 \\
\hline-\frac{3}{4} & -\frac{1}{2} & -\frac{1}{2} & -1 & 0 & 0 & -\frac{1}{2} & -\frac{1}{4} \\
\frac{1}{4} & \frac{1}{4} & 0 & -\frac{3}{4} & 0 & 0 & \frac{1}{2} & 1 \\
\hline 0 & 0 & -1 & \frac{3}{4} & \frac{3}{4} & 0 & 0 & 0 \\
0 & 0 & \frac{3}{4} & -\frac{3}{4} & 0 & 1 & 0 & 0 \\
\hline 0 & 0 & 0 & -\frac{1}{2} & 0 & 0 & -\frac{1}{2} & \frac{1}{4} \\
0 & 0 & 0 & \frac{1}{4} & 0 & 0 & -\frac{1}{2} & -\frac{1}{4}
\end{array}\right]
$$

and

$$
B=\left[\begin{array}{cccc}
B_{1} & 0 & 0 & 0 \\
0 & B_{2} & 0 & 0 \\
0 & 0 & B_{3} & 0 \\
0 & 0 & 0 & B_{4}
\end{array}\right]=\operatorname{diag}\left(\left[\begin{array}{c}
0 \\
-\frac{1}{2}
\end{array}\right],\left[\begin{array}{c}
0 \\
\frac{1}{2}
\end{array}\right],\left[\begin{array}{c}
\frac{1}{2} \\
0
\end{array}\right],\left[\begin{array}{l}
-1 \\
-1
\end{array}\right]\right) .
$$

The dynamics of the overall network are written in compact form as $x(k+1)=A x(k)+B u(k)$, where $x=$ $\operatorname{col}\left(x_{1}, \ldots, x_{4}\right) \in \mathbb{R}^{8}$ and $u=\operatorname{col}\left(u_{1}, \ldots, u_{4}\right) \in \mathbb{R}^{4}$. System (5) is not open-loop stable; some of the eigenvalues of $A$ lie outside the open unit disk $\mathcal{B}:=\{\lambda \in \mathbb{C}|| \lambda \mid<1\}$. Moreover, it can be shown that system 3 is neither openloop asymptotically stable nor stabilizable under decoupled operation, i.e., if $x_{j}(k)$ is zero for all $j \in \mathcal{N}_{3}$ and $k \in \mathbb{Z}_{+}$. Still, it is possible to stabilize system (5) using a static global-state feedback law $u=K x$, for some $K \in \mathbb{R}^{4 \times 8}$, as $A$ and $B$ satisfy the Kalman rank condition for controllability (i.e., $\left.\operatorname{rank}\left[\begin{array}{llll}B & A B & \ldots & A^{7} B\end{array}\right]=8\right)$.

Next, consider the design of such a feedback law for (5). State-of-the-art non-centralized schemes for assessing closed-loop stability usually employ a set of storage functions $\left\{V_{i}(\cdot)\right\}_{i \in \mathcal{I}}$ with $V_{i}\left(x_{i}\right)=x_{i}^{\top} P_{i} x_{i}, P_{i} \in \mathbb{R}^{n_{i} \times n_{i}}$, to construct a Lyapunov candidate $V(x):=\sum_{i \in \mathcal{I}} V_{i}\left(x_{i}\right)=$ $x^{\top} P x$ with block-diagonal $P:=\operatorname{diag}\left(\left\{P_{i}\right\}_{i \in \mathcal{I}}\right) \in \mathbb{R}^{n \times n}$. Note that in many approaches, neither of the functions $V_{i}(\cdot)$ is required to be a $\mathrm{LF}$ for its corresponding local system, see, e.g., (Langbort et al., 2004; Jokić and Lazar, $2009)$. In this case, the above described methods succeed if there exist matrices $P:=\operatorname{diag}\left(\left\{P_{i}\right\}_{i \in \mathbb{Z}_{[1,4]}}\right) \in \mathbb{R}^{8 \times 8}$, $K \in \mathbb{R}^{4 \times 8}$ such that $P \succ 0$ and

$$
(A+B K)^{\top} P(A+B K)-P \prec 0,
$$

in accordance with Thm. 2.2. Verifying the existence of matrices $P, K$ that satisfy the above inequality amounts to solving a semi-definite program $((6)$ can be rewritten into linear form via the Schur complement rule), which is trivial for the small NDS considered here. Using this approach, it can be shown that there is no block-diagonal $P:=$ $\operatorname{diag}\left(\left\{P_{i}\right\}_{i \in \mathbb{Z}_{[1,4]}}\right)$ with $P_{i} \in \mathbb{R}^{2 \times 2}, i \in \mathbb{Z}_{[1,4]}$, that satisfies (6) and, consequently, conventional scalable design of fixed state-feedback controllers fails. Notice that this also impedes the application of decentralized control schemes that rely on a block-diagonal $K$, see, e.g., (Sandell et al., 1978; Šiljak, 1991). The same limitation applies to scalable stabilization techniques that belong to the framework of model predictive control, see, e.g., (Dunbar, 2007; Alessio and Bemporad, 2007; Raimondo et al., 2007). For all these methods, the stability guarantee completely depends on the existence of fixed quadratic storage functions.

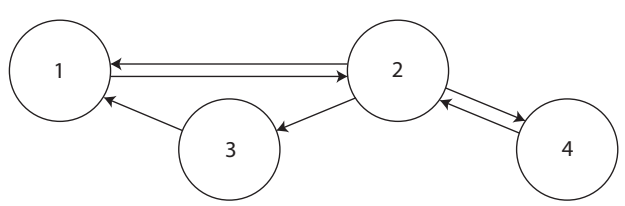

Fig. 1. Topology of the example network.

Motivated by the above observations, the objective of this paper is to provide an alternative scalable stabilization method that is able to stabilize discrete-time NDS (5). This is attained via parameterized storage functions.

\section{MAIN RESULTS}

Let $c_{1} \in \mathbb{R}_{>0}, c_{2} \in \mathbb{R}_{>c_{1}}$ and consider a Lyapunov function with quadratic bounds, i.e., a LF $V: \mathbb{R}^{n} \rightarrow \mathbb{R}_{+}$that satisfies (2a) with $\alpha_{1}(s):=c_{1} s^{2}, \alpha_{2}(s):=c_{2} s^{2}$.

Lemma 4.1. The following two statements are equivalent:

(i) System (4) is GES.

(ii) System (4) admits a LF with quadratic bounds.

Lemma 4.1 is proven in (Jiang and Wang, 2002, Thm. 2), for the general case of a time-varying $V$ and system dynamics. Next, we decentralize this result by showing that every LF with quadratic bounds can be decomposed into a set of local, quadratic storage functions, each parameterized by a full-state-dependent matrix $\widetilde{P}_{i}(x) \in$ $\mathbb{R}^{n_{i} \times n_{i}}$.

Lemma 4.2. Let $V(x)$ be a $L F$ for (4) with quadratic bounds. Then there exist $\widetilde{P}_{i}(x) \in \mathbb{R}^{n_{i} \times n_{i}}, i \in \mathcal{I}$, such that $V(x)=\max _{i \in \mathcal{I}} V_{i}\left(\widetilde{P}_{i}(x), x_{i}\right)$ with $V_{i}\left(\widetilde{P}_{i}(x), x_{i}\right)=$ $x_{i}^{\top} \widetilde{P}_{i}(x) x_{i}$, for all $x_{i} \in \mathbb{R}^{n_{i}}$.

Proof. To prove Lemma 4.2, let us construct functions $V_{i}\left(\widetilde{P}_{i}(x), x_{i}\right)$ that equal $V(x)$ except when $x_{i}$ lies within a particular neighborhood of the origin, whose size is determined by the full network state $x$. Since $\left\|x_{i}\right\|_{2}^{2} \leq$ $\|x\|_{2}^{2}=\sum_{i \in \mathcal{I}}\left\|x_{i}\right\|_{2}^{2} \leq N \max _{i \in \mathcal{I}}\left\|x_{i}\right\|_{2}^{2}$, define for all $x \in \mathbb{R}^{n}$ and $i \in \mathcal{I}$,

$$
\widetilde{P}_{i}(x):= \begin{cases}V(x)\left\|x_{i}\right\|_{2}^{-2} I_{n_{i}}, & \left\|x_{i}\right\|_{2}^{2} \geq \frac{1}{N}\|x\|_{2}^{2}, \\ N V(x)\|x\|_{2}^{-2} I_{n_{i}}, & \left\|x_{i}\right\|_{2}^{2}<\frac{1}{N}\|x\|_{2}^{2} \neq 0, \\ N c_{2} I_{n_{i}}, & \|x\|_{2}^{2}=0 .\end{cases}
$$

From the quadratic bounds on $V(x)$, it follows that $\widetilde{P}_{i}(x)$ is well-defined and $c_{1} I_{n_{i}} \preceq \widetilde{P}_{i}(x) \preceq N c_{2} I_{n_{i}}$ for all $x \in \mathbb{R}^{n}$. Next, we show by contradiction that for any $x \in \mathbb{R}^{n}$, $\left\|x_{i}\right\|_{2}^{2} \geq \frac{1}{N}\|x\|_{2}^{2}$ for at least one $i \in \mathcal{I}$. Suppose that $\left\|x_{i}\right\|_{2}^{2}<\frac{1}{N}\|x\|_{2}^{2}$ for all $i \in \mathcal{I}$. This implies that $\|x\|_{2}^{2}=$ $\sum_{i \in \mathcal{I}}\left\|x_{i}\right\|_{2}^{2}<N \frac{1}{N}\|x\|_{2}^{2}=\|x\|_{2}^{2}$. Since this assumption yields a contradiction, for all $x \in \mathbb{R}^{n}$, there exists a nonempty set $\widetilde{\mathcal{I}} \subseteq \mathcal{I}$ such that $\left\|x_{l}\right\|_{2}^{2} \geq \frac{1}{N}\|x\|_{2}^{2}$ for $l \in \widetilde{\mathcal{I}}$. Now consider the functions $V_{i}: \mathbb{R}^{n_{i} \times n_{i}} \times \mathbb{R}^{n_{i}} \rightarrow \mathbb{R}_{+}$, with $V_{i}\left(\widetilde{P}_{i}(x), x_{i}\right):=x_{i}^{\top} \widetilde{P}_{i}(x) x_{i}$ for all $i \in \mathcal{I}$. From the above result and the construction of $\widetilde{P}_{i}(x)$, it follows that

$$
\max _{i \in \mathcal{I}} V_{i}\left(\widetilde{P}_{i}(x), x_{i}\right)=V_{l}\left(\widetilde{P}_{l}(x), x_{l}\right)=V(x), l \in \widetilde{\mathcal{I}}, x \in \mathbb{R}^{n},
$$

which concludes the proof.

Let $\mathbb{P}_{i}:=\mathbb{R}^{n_{i} \times n_{i}}, i \in \mathcal{I}$, denote a set of real-valued parameter sets/matrices and consider the following definition. 
Definition 4.3. Let $c_{1, i} \in \mathbb{R}_{>0}, c_{2, i} \in \mathbb{R}_{\geq c_{1, i}}$ and $\rho_{i} \in$ $\mathbb{R}_{[0,1)}$ for all $i \in \mathcal{I}$. A set of functions $\left\{V_{i}\right\}_{i \in \mathcal{I}}$ with $V_{i}\left(M_{i}, x_{i}\right):=x_{i}^{\top} M_{i} x_{i}, M_{i} \in \mathbb{P}_{i}:=\mathbb{R}^{n_{i} \times n_{i}}$, that satisfy

$$
\begin{aligned}
& c_{1, i}\left\|x_{i}\right\|_{2}^{2} \leq V_{i}\left(P_{i}(x), x_{i}\right) \leq c_{2, i}\left\|x_{i}\right\|_{2}^{2} \\
& V_{i}\left(P_{i}\left(x^{+}\right), x_{i}^{+}\right) \leq \rho_{i} \max _{j \in \mathcal{I}} V_{j}\left(P_{j}(x), x_{j}\right),
\end{aligned}
$$

for all $x \in \mathbb{R}^{n}, x^{+}=\Phi(x), i \in \mathcal{I}$ and some set of fullstate parameterizations $P_{i}: \mathbb{R}^{n} \rightarrow \mathbb{P}_{i}, i \in \mathcal{I}$, is a set of parameterized quadratic LFs ( $p$-qLFs) for (3).

Corollary 4.4. The following statements are equivalent:

(i) System (4) is GES.

(ii) System (4) admits a set of p-qLFs.

Corrolary 4.4 straightforwardly follows from Lemma 4.1 and Lemma 4.2 , by observing that $V_{i}\left(P_{i}(x), x_{i}\right)$ with $P_{i}(x):=\widetilde{P}_{i}(x)$ as defined by $(7)$ yields p-qLFs that satisfy (8) with $c_{1, i}=c_{1}, c_{2, i}=c_{2}$, and $\rho_{i}=\rho, i \in \mathcal{I}$. Local functions whose maximum defines a LF are frequently used in NDS stability studies, see, e.g., (Dashkovskiy et al., 2010), which deals with establishing input-to-state stability for networked systems that are subject to disturbances. However, in contrast to the method employed in (Dashkovskiy et al., 2010), (8b) requires neither of the functions $V_{i}$ to be a LF for the corresponding local system dynamics, which decreases conservatism considerably.

Next, we propose two non-centralized synthesis solutions to the NDS stabilization problem based on the above results. Firstly, consider a global-state feedback scheme that serves as a starting point for both approaches. For this, consider a non-autonomous NDS with graph $\mathcal{G}=$ $(\mathcal{S}, \mathcal{E})$, and let each system $\varsigma_{i} \in \mathcal{S}$ be governed by the dynamics

$$
x_{i}(k+1)=\psi_{i}\left(x_{i}(k), v_{i}\left(x_{\mathcal{N}_{i}}(k)\right), u_{i}(k)\right), \quad k \in \mathbb{Z}_{+} .
$$

Here, $x_{i}(k) \in \mathbb{R}^{n_{i}}, v_{i}\left(x_{\mathcal{N}_{i}}(k)\right) \in \mathbb{R}^{n_{v_{i}}}$ and $u_{i}(k) \in$ $\mathbb{R}^{m_{i}}$ is the control input to the $i$-th system at time $k$. The functions $\psi_{i}: \mathbb{R}^{n_{i}} \times \mathbb{R}^{n_{v_{i}}} \times \mathbb{R}^{m_{i}} \rightarrow \mathbb{R}^{n_{i}}$ satisfy $\psi_{i}\left(0_{n_{i}}, 0_{n_{v_{i}}}, 0_{m_{i}}\right)=0_{n_{i}}$ for all $i \in \mathcal{I}$.

Given Def. 4.3, let us formulate an optimization control problem to be solved on-line, in a receding horizon fashion, that yields trajectory-dependent p-qLFs. This means that the computed sequences $\left\{P_{i}(x(k))\right\}_{k \in \mathbb{Z}_{+}}$are valid only along the corresponding closed-loop trajectory $\{x(k)\}_{k \in \mathbb{Z}_{+}}$.

Problem 4.5. Centralized $p$-qLF-based synthesis.

At each $k \in \mathbb{Z}_{+}$, let $x(k):=\operatorname{col}\left(\left\{x_{i}(k)\right\}_{i \in \mathcal{I}}\right)$ be known. Let $c_{1, i} \in \mathbb{R}_{>0}, c_{2, i} \in \mathbb{R}_{\geq c_{1, i}}$ and $\rho_{i} \in \mathbb{R}_{[0,1)}, i \in \mathcal{I}$, be given and consider the following inequalities

$$
\begin{gathered}
c_{1, i}\left\|x_{i}(0)\right\|_{2}^{2} \leq V_{i}\left(P_{i}(x(0)), x_{i}(0)\right) \leq c_{2, i}\left\|x_{i}(0)\right\|_{2}^{2} \\
c_{1, i}\left\|x_{i}^{+}(k)\right\|_{2}^{2} \leq V_{i}\left(P_{i}\left(x^{+}(k)\right), x_{i}^{+}(k)\right) \leq c_{2, i}\left\|x_{i}^{+}(k)\right\|_{2}^{2} \\
V_{i}\left(P_{i}\left(x^{+}(k)\right), x_{i}^{+}(k)\right) \leq \rho_{i} \max _{j \in \mathcal{I}} V_{j}\left(P_{j}(x(k)), x_{j}(k)\right),
\end{gathered}
$$

for all $x_{i}^{+}(k)=\psi_{i}\left(x_{i}(k), v_{i}\left(x_{\mathcal{N}_{i}}(k)\right), u_{i}(k)\right), i \in \mathcal{I}$. If $k=0$, find $u_{i}(0) \in \mathbb{R}^{m_{i}}$ and $P_{i}(x(0)), P_{i}\left(x^{+}(0)\right) \in \mathbb{P}_{i}$ that satisfy (10). If $k \in \mathbb{Z}_{\geq 1}$, set $P_{i}(x(k))=P_{i}\left(x^{+}(k-1)\right)$ and find $u_{i}(k) \in \mathbb{R}^{m_{i}}, P_{i}\left(x^{+}(k)\right) \in \mathbb{P}_{i}$ that satisfy (10b)-(10c).

Notice that apart from the $\max _{j \in \mathcal{I}} V_{j}(\cdot)$-term in (10c), conditions (10) concern subsystem parameters only. This allows for non-centralized implementation of Prob. 4.5, by assigning the problem of synthesizing $P_{i}(x(k))$ and $u_{i}(k)$ to network node $i \in \mathcal{I}$, while providing it with knowledge of the $i$-th subsystem model, i.e., (9), state information $\left(x_{i}, v_{i}\left(x_{\mathcal{N}_{i}}\right)\right)$ and the value of $\max _{j \in \mathcal{I}} V_{j}(\cdot)$. The latter can be determined efficiently via multi-branched recursive communication, see (Cormen et al., 2001).

Next, let $\bar{\pi}_{i}: \mathbb{R}^{n} \rightarrow \mathbb{R}^{m_{i}}$ be a feedback law that selects an arbitrary control action $u_{i}$ out of the set of solutions to Prob. 4.5 for each $x \in \mathbb{R}^{n}$. Moreover, let

$$
x_{i}(k+1)=\bar{\phi}_{i}(x(k)), \quad k \in \mathbb{Z}_{+},
$$

with $\bar{\phi}_{i}(x):=\psi_{i}\left(x_{i}, v_{i}\left(x_{\mathcal{N}_{i}}\right), \bar{\pi}_{i}(x)\right)$, describe system $(9)$ in closed-loop with global-state feedback law $\bar{\pi}_{i}$.

Theorem 4.6. Let $\mathbb{X} \subset \mathbb{R}^{n}$ with $0_{n} \in \operatorname{int}(\mathbb{X})$ be bounded and such that Prob. 4.5 is recursively feasible for all $x(0)=\operatorname{col}\left(\left\{x_{i}(0)\right\}_{i \in \mathcal{I}}\right) \in \mathbb{X}$. Then, (11) is $E S(\mathbb{X})$.

Proof. Feasibility of Prob. 4.5 for all $x_{0}:=x(0) \in \mathbb{X}$ and corresponding $x(k) \in \mathbb{R}^{n}, k \in \mathbb{Z}_{+}$, allows for recursive application of $(10 \mathrm{c})$, which yields

$$
\begin{aligned}
& V(P(x(k)), x(k)) \\
& \quad:=\max _{i \in \mathcal{I}} V_{i}\left(P_{i}(x(k)), x_{i}(k)\right) \leq \bar{\rho}^{k} V\left(P\left(x_{0}\right), x_{0}\right),
\end{aligned}
$$

where $\bar{\rho}:=\max _{i \in \mathcal{I}} \rho_{i}$ and $P(x):=\left\{P_{i}(x)\right\}_{i \in \mathcal{I}}$. Then, (10a)-(10b) yield

$$
\begin{aligned}
& V(P(x(k)), x(k)) \geq \frac{1}{N} \sum_{i \in \mathcal{I}} c_{1, i}\left\|x_{i}(k)\right\|_{2}^{2} \geq \frac{c_{1}}{N}\|x(k)\|_{2}^{2} \\
& V(P(x(k)), x(k)) \leq \max _{i \in \mathcal{I}}\left(c_{2, i}\left\|x_{i}(k)\right\|_{2}^{2}\right) \leq \bar{c}_{2, i}\|x(k)\|_{2}^{2},
\end{aligned}
$$

where $\underline{c}_{1}:=\min _{i \in \mathcal{I}} c_{1, i}$ and $\bar{c}_{2}:=\max _{i \in \mathcal{I}} c_{2, i}$. Therefore $\|x(k)\|_{2}^{2} \leq \bar{\rho}^{k} \frac{N \bar{c}_{2}}{\underline{c}_{1}}\|x(0)\|_{2}^{2}$, for all $x(0) \in \mathbb{X}$ and $k \in \mathbb{Z}_{+}$. Hence, $(11)$ is $\operatorname{ES}(\mathbb{X})$ with $\mu:=\sqrt{\bar{\rho}}$ and $c:=\sqrt{\frac{N \bar{c}_{2}}{\underline{c}_{1}}}$.

Even though methods for evaluating $\max _{j \in \mathcal{I}} V_{j}(\cdot)$ in an efficient, distributed fashion exist, the corresponding extent of communication might still become infeasible for certain large-scale applications. In what follows, we will therefore propose a way to further decentralize the parameterized stabilization problem, by generating $V_{i}\left(P_{i}(x(k)), x_{i}(k)\right)$ and $u_{i}(k)$ based on local information only.

Let $\left\{J_{i}(\cdot)\right\}_{i \in \mathcal{I}}$ with $J_{i}\left(\lambda_{i}\right):=\lambda_{i}$ for $\lambda_{i} \in \mathbb{R}_{+}$be a set of cost functions. Now consider the following problem.

Problem 4.7. Almost-decentralized p-qLF-based synthesis. Consider Prob. 4.5, but replace (10c) by

$$
\begin{gathered}
V_{i}\left(P_{i}\left(x^{+}(k)\right), x_{i}^{+}(k)\right) \leq \\
\rho_{i} \max _{j \in \overline{\mathcal{N}}_{i}} V_{j}\left(P_{j}(x(k)), x_{j}(k)\right)+\lambda_{i}(k) \\
\lambda_{i}(k) \geq 0,
\end{gathered}
$$

for all $x_{i}^{+}(k)=\psi_{i}\left(x_{i}(k), v_{i}\left(x_{\mathcal{N}_{i}}(k)\right), u_{i}(k)\right), i \in \mathcal{I}$. If $k=0$, minimize $J_{i}\left(\lambda_{i}(0)\right)$ over $u_{i}(0) \in \mathbb{R}^{m_{i}}$, $P_{i}(x(0)), P_{i}\left(x^{+}(0)\right) \in \mathbb{P}_{i}$ and $\lambda_{i}(0)$ such that $(10 \mathrm{a})-(10 \mathrm{~b})$ and (12) hold. If $k \in \mathbb{Z}_{\geq 1}$, set $P_{i}(x(k))=P_{i}\left(x^{+}(k-1)\right)$ and minimize $J_{i}\left(\lambda_{i}(k)\right)$ over $u_{i}(k) \in \mathbb{R}^{m_{i}}, P_{i}\left(x^{+}(k)\right) \in \mathbb{P}_{i}$ and $\lambda_{i}(k)$ such that (10b) and (12) hold.

Note that in Prob. 4.7, only subsystem model (9) and the values of $\left(x_{i}, v_{i}\left(x_{\mathcal{N}_{i}}\right)\right)$ and $\max _{j \in \overline{\mathcal{N}}_{i}} V_{j}(\cdot)$ are needed to synthesize $P_{i}(x(k)), u_{i}(k)$ and $\lambda_{i}(k)$. From $\overline{\mathcal{N}}_{i}:=\mathcal{N}_{i} \cup\{i\}$, it follows that a single run of information exchange among direct neighbors is sufficient to acquire this knowledge.

Next, let $\pi_{i}: \mathbb{R}^{n_{i}} \times \mathbb{R}^{n_{v_{i}}} \rightarrow \mathbb{R}^{m_{i}}$ be a feedback law that selects an arbitrary control action $u_{i}$ from the solution set of Prob. 4.7 for each $\left(x_{i}, v_{i}\left(x_{\mathcal{N}_{i}}\right)\right) \in \mathbb{R}^{n_{i}} \times \mathbb{R}^{n_{v_{i}}}$. Since 
$\lambda_{i}(k)$ is unbounded from above for all $k \in \mathbb{Z}_{+}$, Prob. 4.7 is recursively feasible and $\pi_{i}$ is well-defined for all $i \in \mathcal{I}$. Let

$$
x_{i}(k+1)=\phi_{i}\left(x_{i}(k), v_{i}\left(x_{\mathcal{N}_{i}}(k)\right)\right)
$$

with $\phi_{i}\left(x_{i}, v_{i}\left(x_{\mathcal{N}_{i}}\right)\right):=\psi_{i}\left(x_{i}, v_{i}\left(x_{\mathcal{N}_{i}}\right), \pi_{i}\left(x_{i}, v_{i}\left(x_{\mathcal{N}_{i}}\right)\right)\right)$, denote the difference equation corresponding to (9) in closed-loop with local-state feedback law $\pi_{i}$. Let $\lambda_{i}^{*}(k)$ denote the $\lambda_{i}$ that optimizes Prob. 4.7 at instant $k \in \mathbb{Z}_{+}$.

Now consider the following theorem.

Theorem 4.8. Let $\mathbb{X}_{D} \subseteq \mathbb{X} \subset \mathbb{R}^{n}$, with $0_{n} \in \operatorname{int}\left(\mathbb{X}_{D}\right)$, be the set of initial conditions $x_{0}:=x(0)$ such that all corresponding trajectories of (13) satisfy $x(k) \in \mathbb{X}$, for all $k \in \mathbb{Z}_{+}$, and let the assumptions on $\mathbb{X}$ given in Thm. 4.6 hold. Then, (13) is $E S\left(\mathbb{X}_{D}\right)$.

Proof. First, we relate Prob. 4.7 to Prob. 4.5. Observe that recursive feasibility of Prob. 4.5 for all $x(0) \in \mathbb{X}$ yields point-wise feasibility of (10b)-(10c) for all $x(k) \in \mathbb{X}$, independent of $k \in \mathbb{Z}_{+}$. Thus, given $x(k) \in \mathbb{X}$ and $P_{i}(x(k)) \in \mathbb{P}_{i}$, there exist $\bar{u}_{i}(k) \in \mathbb{R}^{m_{i}}$ and $\bar{P}_{i}\left(x^{+}(k)\right) \in \mathbb{P}_{i}$, $i \in \mathcal{I}$, that satisfy (10b)-(10c). Now consider the function $\Delta_{i}: \mathbb{P}_{1} \times \ldots \times \mathbb{P}_{N} \times \mathbb{R}^{n}$, with

$\Delta_{i}(P(x), x):=$

$$
\rho_{i}\left(\max _{j \in \mathcal{I}} V_{j}\left(P_{j}(x), x_{j}\right)-\max _{j \in \overline{\mathcal{N}}_{i}} V_{j}\left(P_{j}(x), x_{j}\right)\right) .
$$

Due to feasibility of (10c) for $x(k) \in \mathbb{X}$ and $P_{i}(x(k)) \in$ $\mathbb{P}_{i}, k \in \mathbb{Z}_{+}$, there is a $\lambda_{i}(k)$ such that $0 \leq \lambda_{i}(k) \leq$ $\Delta_{i}(P(x(k)), x(k))$, that satisfies (12) for $\bar{P}_{i}\left(\bar{x}^{+}(k)\right)$ and $\bar{u}_{i}(k)$, and yields $x^{+}(k) \in \mathbb{X}$ if $x(0) \in \mathbb{X}_{\mathrm{D}}$. By construction,

$0 \leq \Delta_{i}(P(x(k)), x(k)) \leq \rho_{i} \max _{j \in \mathcal{I}} V_{j}\left(P_{j}(x(k)), x_{j}(k)\right)$ for all $P_{i}(x(k)) \in \mathbb{P}_{i}, x(k) \in \mathbb{R}^{n}$. Note that the term $\max _{j \in \mathcal{I}} V_{j}\left(P_{j}(x), x_{j}\right)$ is bounded on bounded sets.

Next, we prove that (13) is $\operatorname{ES}\left(\mathbb{X}_{\mathrm{D}}\right)$. Minimization of $J_{i}\left(\lambda_{i}(k)\right)$ over $\lambda_{i}(k)$ yields $0 \leq \lambda_{i}^{*}(k) \leq \Delta_{i}(P(x(k)), x(k))$ and consequently

$$
V_{i}\left(P_{i}\left(x^{+}(k)\right), x_{i}^{+}(k)\right) \leq \rho_{i} \max _{j \in \mathcal{I}} V_{j}\left(P_{j}(x(k)), x_{j}(k)\right)
$$

for all $x_{i}^{+}(k) \in \phi\left(x_{i}(k), v_{i}\left(x_{\mathcal{N}_{i}}(k)\right)\right), i \in \mathcal{I}$ and $k \in \mathbb{Z}_{+}$. Then, exponential stability of (13) in $\mathbb{X}_{\mathrm{D}}$ straightforwardly follows along the lines of the proof of Thm. 4.6.

In contrast to (10), conditions (10a)-(10b) and (12) concern local/neighboring system parameters only. Hence, we have obtained a non-centralized control scheme that guarantees closed-loop stability while relying on non-iterative communication among directly connected systems only.

\subsection{Implementation via semi-definite programming}

Next, we show that for input-affine NDS and parameterized quadratic LFs, Prob. 4.7 can be formulated as a set of low-complexity semi-definite programming problems. Consider NDS dynamics (9) with

$\psi_{i}\left(x_{i}, v_{i}\left(x_{\mathcal{N}_{i}}\right), u_{i}\right):=f_{i}\left(x_{i}, v_{i}\left(x_{\mathcal{N}_{i}}\right)\right)+g_{i}\left(x_{i}, v_{i}\left(x_{\mathcal{N}_{i}}\right)\right) u_{i}$,

for $i \in \mathcal{I}$, where $x_{i} \in \mathbb{R}^{n_{i}}, u_{i} \in \mathbb{R}^{m_{i}}, f_{i}: \mathbb{R}^{n_{i}} \times \mathbb{R}^{n_{v_{i}}} \rightarrow \mathbb{R}^{n_{i}}$, $g_{i}: \mathbb{R}^{n_{i}} \times \mathbb{R}^{n_{v_{i}}} \rightarrow \mathbb{R}^{n_{i} \times m_{i}}$, such that $f_{i}\left(0_{n_{i}}, 0_{n_{v_{i}}}\right)=0_{n_{i}}$. Let $\gamma_{i} \in \mathbb{R}_{>0}, \Gamma_{i} \in \mathbb{R}_{\geq \gamma_{i}}$ and let $P_{i}(x(0))=\Gamma_{i} I_{n_{i}}$. Now consider the matrix inequalities

$$
\begin{aligned}
& Z_{i}(k) \succeq \Gamma_{i}^{-1} I_{n_{i}}, \quad Z_{i}(k) \preceq \gamma_{i}^{-1} I_{n_{i}}, \quad \lambda_{i}(k) \geq 0 \\
& {\left[\begin{array}{cc}
\rho_{i} \max _{j \in \overline{\mathcal{N}}_{i}} x_{j}(k)^{\top} P_{j}(x(k)) x_{j}(k)+\lambda_{i}(k) & * \\
\widetilde{f}_{i}(x(k))+\widetilde{g}_{i}(x(k)) u_{i}(k) & Z_{i}(k)
\end{array}\right] \succeq 0,}
\end{aligned}
$$

where $\widetilde{f}_{i}(x):=f_{i}\left(x_{i}, v_{i}\left(x_{\mathcal{N}_{i}}\right)\right)$ and $\widetilde{g}_{i}(x):=g_{i}\left(x_{i}, v_{i}\left(x_{\mathcal{N}_{i}}\right)\right)$. Lemma 4.9. At time $k \in \mathbb{Z}_{+}$and node $i \in \mathcal{I}$, let $x_{i}(k)$, $x_{\mathcal{N}_{i}}(k), \rho_{i}, \gamma_{i}, \Gamma_{i}$ and $\left\{P_{j}(x(k))\right\}_{j \in \overline{\mathcal{N}}_{i}}$ be given. Suppose that $\left\{Z_{i}(k), u_{i}(k), \lambda_{i}(k)\right\}$ is a solution of (15). Then $P_{i}\left(x^{+}(k)\right)=Z_{i}(k)^{-1}, u_{i}(k)$ and $\lambda_{i}(k)$ is a solution of $(10 \mathrm{~b})$ and (12) with $c_{1, i}:=\gamma_{i}$ and $c_{2, i}:=\Gamma_{i}$.

Proof. As $P_{i}(x(0))=\Gamma_{i} I_{n_{i}}$, (10a) follows with $c_{1, i}:=\gamma_{i}$ and $c_{2, i}:=\Gamma_{i}$. Also, the Schur complement of (15b) gives

$$
\begin{aligned}
& \rho_{i} \max _{j \in \overline{\mathcal{N}}_{i}} x_{j}^{\top} P_{j}(x) x_{j}+\lambda_{i} \\
& \quad-\left(\widetilde{f}_{i}(x)+\widetilde{g}_{i}(x) u_{i}\right)^{\top} Z_{i}^{-1}\left(\widetilde{f}_{i}(x)+\widetilde{g}_{i}(x) u_{i}\right) \geq 0,
\end{aligned}
$$

where the time-dependence was omitted for brevity. Combining (14) and (16) yields (12a) with $P_{i}\left(x^{+}(k)\right)=$ $Z_{i}(k)^{-1}$. Moreover, (15a) yields $(12 \mathrm{~b})$ and $\gamma_{i} I_{n_{i}} \preceq$ $Z_{i}(k)^{-1}=P_{i}\left(x^{+}(k)\right) \preceq \Gamma_{i} I_{n_{i}}$, which is (10b).

Lemma 4.9 provides a scalable SDP-based receding horizon control scheme for input-affine NDS. The resulting set of local control laws is stabilizing under recursive feasibility of Prob. 4.5, or, under recursive optimality with respect to linear cost functions $J_{i}\left(\lambda_{i}\right)$ if Prob. 4.7 is considered.

\section{SIMULATION RESULTS}

Next, p-qLF-based controller synthesis is illustrated for the network of linear time-invariant dynamical systems described in Sect. 3, using the SDP-based implementation given in Sect. 4.1. Network (5) is described by (14) with $f_{i}\left(x_{i}, v_{i}\left(x_{\mathcal{N}_{i}}\right)\right):=A_{i i} x_{i}+\sum_{j \in \mathcal{N}_{i}} A_{i j} x_{j}$ and $g_{i}\left(x_{i}, v_{i}\left(x_{\mathcal{N}_{i}}\right)\right):=B_{i}$, for $i \in \mathbb{Z}_{[1,4]}$. In the simulations, the values $\gamma_{i}=0.1, \Gamma_{i}=1$ and $\rho_{i}=0.95$ were used for all $i \in \mathbb{Z}_{[1,4]}$. Fig. 2 shows the trajectories of $x(k), u_{i}(k)$ and $\lambda_{i}(k)$ as generated by Prob. 4.7 in closed-loop with (5) for initial condition $x(0)=$

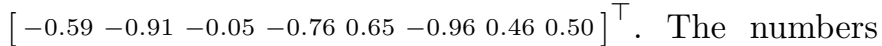
in the $\lambda_{i}(k)$ plot denote the index of the "dominant subsystem", i.e., $\arg \max _{i \in \mathbb{Z}_{[1,4]}} V_{i}\left(P_{i}(x(k)), x_{i}(k)\right)$ at discretetime instants $k \in \mathbb{Z}_{[0,15]}$. The corresponding evolution of $V_{i}\left(P_{i}(x(k)), x_{i}(k)\right)$ and $V(P(x(k)), x(k)):=\max _{i \in \mathbb{Z}_{[1,4]}}$ $V_{i}\left(P_{i}(x(k)), x_{i}(k)\right)$ is depicted in Fig. 3. Therein, it can be observed that even though neither of the p-qLFs $V_{i}(\cdot)$ is enforced to decrease monotonically, the corresponding Lyapunov function for the full network $V(\cdot)$ does, which in turn results in asymptotically converging state trajectories. Also, note that $\lambda_{3}(k) \neq 0$ for $k \in\{4,9\}$, to relax convergence condition (12a) on $V_{3}(\cdot)$ as $\arg \max _{j \in \mathbb{Z}_{[1,4]}} V_{j}\left(P_{j}(x(k)), x_{j}(k)\right)=1 \notin \overline{\mathcal{N}}_{3}$ at these time instants. Consequently, the control problem remains feasible and asymptotically converging closedloop state trajectories are obtained even though each controller employs local information only. Fig. 4 shows the level sets of $V_{i}\left(P_{i}(x(k)), x_{i}(k)\right), i \in \mathbb{Z}_{[1,4]}$, i.e., $\{z \in$ $\left.\mathbb{R}^{2} \mid V_{i}\left(P_{i}(x(k)), z\right)=\frac{1}{2}\right\}_{k \in \mathbb{Z}_{[0,15]}}$. Note that the change in $P_{4}(x(k))$, corresponding to the sparsely-connected system 4 , is rather small. Still, the strong variation of the level sets for the other systems shows that in this simulation, the flexibility associated with $\mathrm{p}-\mathrm{qLF}$ parameterization is amply exploited by controllers 1-3. This enabled synthesis of stabilizing control actions, whereas standard Lyapunov techniques that rely on fixed storage functions failed. 

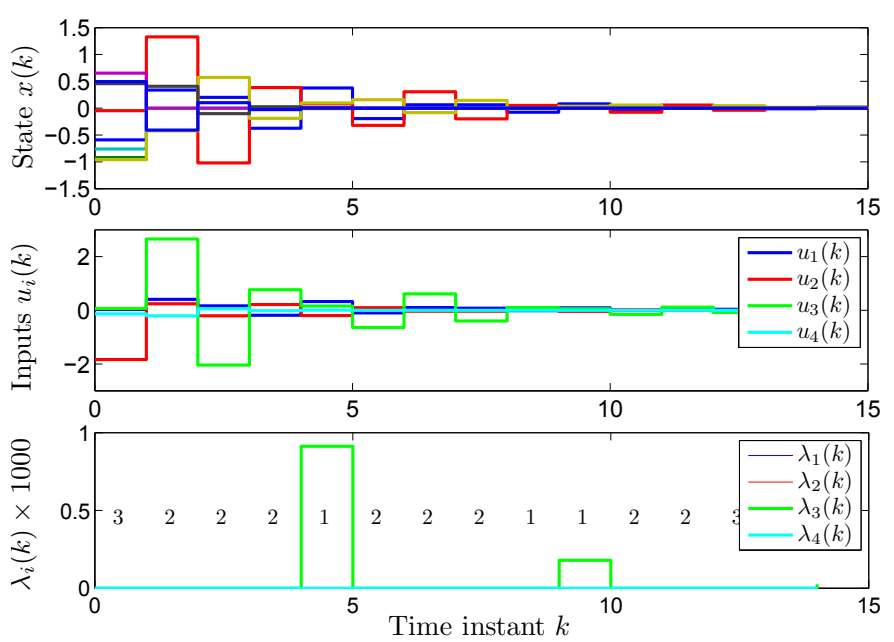

Fig. 2. Simulated state, input and $\lambda_{i}$ trajectories.

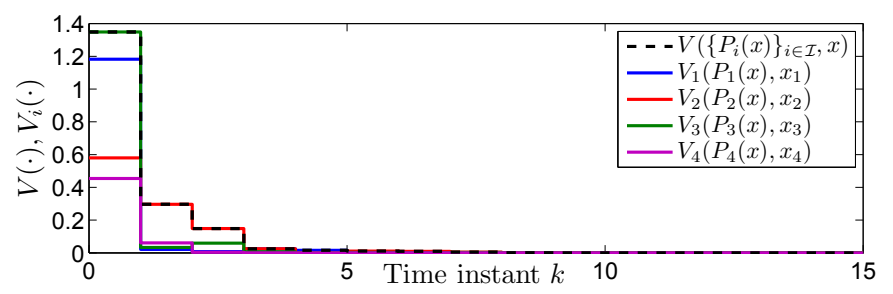

Fig. 3. Simulated evolution of $V_{i}(\cdot)$ and $V(\cdot)$ over time.
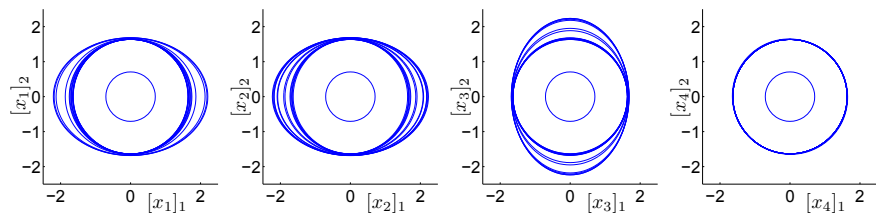

Fig. 4. Level sets of $V_{i}\left(P_{i}(x(k)), x_{i}(k)\right)$ for $k \in \mathbb{Z}_{[0,15]}$.

\section{CONCLUSIONS}

This paper addressed the problem of stabilizing networked dynamical systems in a scalable way. As a first contribution, an example was provided to illustrate that standard NDS stabilization methods can fail even for simple linear time-invariant systems. Then, a solution to this issue was proposed, by decentralizing the controller synthesis via a set of parameterized local functions. The employed stability conditions allow for max-type construction of a Lyapunov function for the closed-loop system, while neither of the parameterized functions is required to be a local LF. It was shown that the provided approach is non-conservative, in the sense that it can find a stabilizing control law for the motivating example network, whereas state-of-the-art noncentralized Lyapunov techniques failed. For input-affine NDS and quadratic parameterized functions, the control scheme was formulated as a set of low-complexity semidefinite programs that are solved in a receding horizon manner.

\section{ACKNOWLEDGEMENT}

This research is supported by SenterNovem/the Dutch Ministry of Economic Affairs and the Veni grant "Flexible Lyapunov Functions for Real-time Control", grant no. 10230, awarded by STW and NWO.

\section{REFERENCES}

Alessio, A. and Bemporad, A. (2007). Decentralized model predictive control of constrained linear systems. 9th European Control Conference, 2813-2818.

Camponogara, E., Jia, D., Krogh, B.H., and Talukdar, S. (2002). Distributed model predictive control. IEEE Control Systems Magazine, 22(1), 44-52.

Cormen, T.H., Leiserson, C.E., Rivest, R.L., and Stein, C. (2001). Introduction to Algorithms. The MIT Press, Cambridge, MA, USA.

Dashkovskiy, S.N., Rüffer, B.S., and Wirth, F.R. (2010). Small gain theorems for large scale systems and construction of ISS Lyapunov functions. SIAM Journal on Control and Optimization, 48(6), 4089-4118.

Dunbar, W. (2007). Distributed receding horizon control of dynamically coupled nonlinear systems. IEEE Transactions on Automatic Control, 52(7), 1249-1263.

Hermans, R.M., Lazar, M., and Jokić, A. (2010). Almost decentralized Lyapunov-based nonlinear model predictive control. In American Control Conference, 39323938. Baltimore, MD, USA.

Jiang, Z.P. and Wang, Y. (2001). Input-to-state stability for discrete-time nonlinear systems. Automatica, 37, 857-869.

Jiang, Z.P. and Wang, Y. (2002). A converse Lyapunov theorem for discrete-time systems with disturbances. Systems \& Control Letters, 45(1), 49-58.

Jokić, A. and Lazar, M. (2009). On decentralized stabilization of discrete-time nonlinear systems. In American Control Conference, 5777-5782. St. Louis, MO, USA.

Langbort, C., Chandra, R.S., and D'Andrea, R. (2004). Distributed control design for systems interconnected over an arbitrary graph. IEEE Transactions on Automatic Control, 49(9), 1502-1519.

Lazar, M. (2006). Model Predictive Control of Hybrid Systems: Stability and Robustness. Ph.D. thesis, Eindhoven University of Technology, Eindhoven, the Netherlands.

Negenborn, R.R., De Schutter, B., and Hellendoorn, J. (2008). Multi-agent model predictive control for transportation networks: Serial versus parallel schemes. Engineering Applications of Artificial Intelligence, 21(3), $353-366$.

Raimondo, D.M., Magni, L., and Scattolini, R. (2007). Decentralized MPC of nonlinear systems: An inputto-state stability approach. International Journal of Robust and Nonlinear Control, 17(17), 1651-1667.

Sandell, N.R., Varaiya, P., Athans, M., and Safonov, M.G. (1978). Survey of decentralized control methods for large scale systems. IEEE Transactions on Automatic Control, 23(2), 108-128.

Strogatz, S.H. (2001). Exploring complex networks. $\mathrm{Na}$ ture, 410, 268-276.

Venkat, A.N., Hiskens, I.A., Rawlings, J.B., and Wright, S.J. (2008). Distributed MPC strategies with application to power system automatic generation control. IEEE Transactions on Control Systems Technology, 16(6), 1192-1206.

Šiljak, D.D. (1991). Decentralized Control of Complex Systems, volume 184 of Mathematics in Science and Engineering. Academic Press, Boston, MA, USA.

Willems, J.C. (1972). Dissipative dynamical systems. Archive for Rational Mechanics and Analysis, 45, 321393. 